\title{
An online version of Rota's basis conjecture
}

\author{
Guus P. Bollen · Jan Draisma
}

Received: 31 March 2014 / Accepted: 3 October 2014 / Published online: 21 October 2014

(C) Springer Science+Business Media New York 2014

\begin{abstract}
Rota's basis conjecture states that in any square array of vectors whose rows are bases of a fixed vector space the vectors can be rearranged within their rows in such a way that afterwards not only the rows are bases, but also the columns. We discuss an online version of this conjecture, in which the permutation used for rearranging the vectors in a given row must be determined without knowledge of the vectors further down the array. The paper contains surprises both for those who believe this online basis conjecture at first glance, and for those who disbelieve it.
\end{abstract}

Keywords Rota's basis conjecture · Exterior algebra · Online algorithms

\section{Background}

Fix a field $K$ and $n \in \mathbb{N}$. The following conjecture first appeared in [12].

Conjecture 1.1 (Rota's basis conjecture, $\left.R B C_{n}(K)\right)$ Let $V=K^{n}$ and let $v=\left(v_{i j}\right)_{i j}$ be an $n \times n$-array of vectors in $V$. Suppose that for all $i=1, \ldots, n$ the $i$-th row $\left(v_{i, 1}, \ldots, v_{i, n}\right)$ of $v$ is a basis of $V$. Then there exist permutations $\pi_{1}, \ldots, \pi_{n} \in S_{n}$ such that for all $j=1, \ldots, n$ the vectors $v_{1, \pi_{1}(j)}, \ldots, v_{n, \pi_{n}(j)}$ form a basis, as well.

Here the vectors $v_{1, \pi_{1}(j)}, \ldots, v_{n, \pi_{n}(j)}$ form the $j$-th column of the array obtained from $v$ by applying, for each $i=1, \ldots, n$, the $i$-th permutation $\pi_{i}$ to the $i$-th row. RBC has a natural generalisation to arbitrary matroids, and there is quite some literature on

\footnotetext{
G. P. Bollen · J. Draisma ( $\square)$

Department of Mathematics and Computer Science, Technische Universiteit Eindhoven, P.O. Box 513, 5600 MB Eindhoven, The Netherlands

e-mail: j.draisma@tue.nl

G. P. Bollen

e-mail: g.p.bollen@tue.nl
} 
this generalisation: e.g. it is true in rank up to three [4], for paving matroids [9], and a version for $k \times n$-arrays with $n>\left(\begin{array}{c}k+1 \\ 2\end{array}\right)$ was proved in [10]. In this paper, however, we restrict ourselves to the vector space version except for a brief remark in the last section.

It is well known that $\mathrm{RBC}_{n}$ is related to a conjecture by Alon and Tarsi on Latin squares. Recall that a Latin square of order $n$ is an $n \times n$-array $a=\left(a_{i j}\right)_{i j}$ with all $a_{i j} \in[n]:=\{1, \ldots, n\}$ such that every element of $[n]$ occurs precisely once in each row and each column. The map $j \mapsto a_{i j}$ is then an element of the symmetric group $S_{n}$ for each fixed $i$, and so is the map $i \mapsto a_{i j}$ for each fixed $j$ and the sign of $a$ is by definition the product of the signs of these $2 n$ permutations. A Latin square is called even if its sign is 1 and odd if its sign is -1 . The number of even Latin squares is denoted as $\operatorname{ELS}(n)$, and the number of odd Latin squares is denoted as $\operatorname{OLS}(n)$. If $n$ is odd and $>2$, then interchanging rows 1 and 2 gives a sign-reversing involution on the set of Latin squares, which proves that $\operatorname{ELS}(n)=\mathrm{OLS}(n)$. The following conjecture from [2] on the case of even $n$ has various reformulations [13].

Conjecture 1.2 (Alon and Tarsi's Latin square conjecture, ATLSC ${ }_{n}$ ) If $n$ is even, then $\operatorname{ELS}(n) \neq \operatorname{OLS}(n)$.

The known relation between $\operatorname{RBC}_{n}(K)$ and $\mathrm{ATLSC}_{n}$ is the following, and has been proved by various authors $[12,14,15]$.

Theorem 1.3 If the characteristic of $K$ does not divide $\operatorname{ELS}(n)-\operatorname{OLS}(n)$, then $\mathrm{RBC}_{n}(K)$ holds. In particular, if the characteristic of $K$ is zero or sufficiently large, then $\mathrm{ATLSC}_{n}$ implies $\mathrm{RBC}_{n}(K)$.

$\mathrm{ATLSC}_{n}$ has been proved for $n$ of the form $p+1$ [6] and for $n$ of the form $p-1$ [11], both with $p$ an odd prime (see also [3] for easy, self-contained proofs). The paper [16] contains a claim to the effect that $\mathrm{ATLSC}_{m}$ implies $\mathrm{ATLSC}_{2 m}$. As pointed out in [11], the proof is incorrect. However, in Sect. 3 we show that $\mathrm{ATLSC}_{m}$ does imply (an online strengthening of) a variant of RBC for $(m+1) \times n$-arrays of vectors with $n>m$ a multiple of $m$.

There is a generalisation of ATLSC in which one counts only the (signs of the) Latin squares whose diagonal entries are fixed to be, say, $n$ [16]. While for even $n$ this generalised conjecture is equivalent to $\mathrm{ATLSC}_{n}$, the generalised conjecture is also meaningful for odd $n$, and was proved in [7] for $n=p$ prime. In Sect. 4 we establish a connection to a version of $\mathrm{RBC}_{n}$ where all bases share a common vector. This is different from the result of [1], which links a variant of ATLSC to a different weakening of RBC.

\section{Online version, main results}

This paper concerns the following natural strengthening of $\operatorname{RBC}_{n}(K)$.

Question 2.1 (Online version of Rota's basis conjecture, $\left.\mathrm{OBC}_{n}(K)\right)$ Does there exist an algorithm for finding the permutations $\pi_{i}$ in $\operatorname{RBC}_{n}(K)$ which is online in the following sense? The rows $\left(v_{1 j}\right)_{j},\left(v_{2 j}\right)_{j}, \ldots,\left(v_{n j}\right)_{j}$ of $v$ are given sequentially to 
the algorithm, and directly after reading the $i$-th row the algorithm fixes permutation $\pi_{i}$, without knowledge of the remaining $n-i$ rows.

For maximum effect, we suggest that the reader takes a few minutes to determine his or her own position on this conjecture. Here are some considerations s/he might take into account: certainly $\pi_{1}$ can be fixed in any manner, and then the second row can be adapted to the first, so $\mathrm{OBC}_{2}(K)$ is equivalent to $\mathrm{RBC}_{2}(K)$-and true, of course. Also, the online problem is reminiscent of a very easy instance of the problem of completing a partially filled Latin square: indeed, if all the bases given to the algorithm are equal to the standard basis $e_{1}, \ldots, e_{n}$ of $V=K^{n}$, then it does not matter how $\pi_{1}, \ldots, \pi_{i}$ are chosen, as long as they do not put twice the same standard basis vector in any column. The $i \times n$ rectangle thus obtained can always be completed to a Latin square; one way to see this is to use the known fact that a regular bipartite graph with non-empty set of edges always contains a perfect matching. On the negative side, with more general vectors than just standard basis vectors one can run into trouble, e.g. as follows. In this $2 \times 4$-array of vectors in $V$ :

$$
\begin{array}{llll}
v_{11}=e_{1} & v_{12}=e_{2} & v_{13}=e_{3} & v_{14}=e_{4} \\
v_{21}=e_{2} & v_{22}=e_{1}+e_{2} & v_{23}=e_{1}+e_{3} & v_{24}=e_{1}+e_{4}
\end{array}
$$

the two rows are bases, and the four column spaces

$$
V_{1}:=\left\langle e_{1}, e_{2}\right\rangle, \quad V_{2}:=\left\langle e_{2}, e_{1}+e_{2}\right\rangle, \quad V_{3}:=\left\langle e_{3}, e_{1}+e_{3}\right\rangle, \quad V_{4}:=\left\langle e_{4}, e_{1}+e_{4}\right\rangle
$$

span two-dimensional spaces, which however all intersect in $\left\langle e_{1}\right\rangle$. Hence any online algorithm would have to avoid this arrangement, because the next, third basis might contain the vector $e_{1}$. More generally, an online algorithm should avoid any $i \times n$-arrangement where the intersection of $\ell$ of the $i$-dimensional column spaces $V_{1}, \ldots, V_{n}$ constructed so far has dimension strictly greater than $n-\ell$. Indeed, if $U:=V_{1} \cap \cdots \cap V_{\ell}$ has dimension $>n-\ell$, then an $(i+1)$-st basis of $V$ containing $>n-\ell$ elements of $U$ cannot be matched with $V_{1}, \ldots, V_{n}$. Conversely, if no $\ell$-intersection has dimension $>n-\ell$, Hall's marriage theorem ensures that any $(i+1)$-st basis can be matched. One might try and make these "general position" conditions more strict and dependent on $i$, so that given any tuple of $i$-dimensional spaces $V_{1}, \ldots, V_{n}$ satisfying the conditions for $i$ and given any basis $v_{1}, \ldots, v_{n}$, a permutation $\pi$ exists such that $V_{1}+\left\langle v_{\pi(1)}\right\rangle, \ldots, V_{n}+\left\langle v_{\pi(n)}\right\rangle$ are all $(i+1)$-dimensional and satisfy the next set of conditions. But very quickly these conditions seem to grow rather intricate, and in Sect. 5 we will see that in fact they cannot be formulated purely in matroidal terms.

By now the reader may have formed his or her own opinion about the answer to $\mathrm{OBC}_{n}$. Ready for our results? Here we go!

Theorem 2.2 If the characteristic of $K$ does not divide $\operatorname{ELS}(n)-\operatorname{OLS}(n)$, then $\mathrm{OBC}_{n}(K)$ holds.

In particular, in the cases where $\mathrm{RBC}_{n}(K)$ holds as a consequence of $\mathrm{ATLSC}_{n}$ (hence with $n$ even), in fact the stronger statement $\mathrm{OBC}_{n}(K)$ holds. 
Theorem 2.3 For any odd $n>2$ and any field $K$ that contains a primitive $m$-th root of unity for all odd $m \leq n, \mathrm{OBC}_{n}(K)$ is false.

Consequently, while $\mathrm{OBC}_{n}(\mathbb{C})$ is true for infinitely many and conjecturally all even $n$, it is false for all odd $n$ except the trivial case $n=1$. We were quite surprised by this dichotomy between even $n$ and odd $n$. In the subsequent two sections of this paper we prove Theorems 2.2 and 2.3, respectively, and discuss some variants for other shapes of arrays. We conclude with some further remarks in Sect. 5.

\section{Even dimensions}

In this section we prove Theorem 2.2. This implies Theorem 1.3, and in fact our contribution may be interpreted as giving more "semantics" to existing proofs of the latter. Since the validity of $\mathrm{OBC}_{n}(K)$ for a field $K$ implies its validity over any subfield of $K$, we assume that $K$ is algebraically closed. We will use algebro-geometric terminology such as hypersurface for a variety in some vector space over $K$ defined by the vanishing of a single non-constant polynomial. We write $\bigwedge^{k} V$ for $k$-th exterior power of $V$ which is the quotient of the $k$-fold tensor power $V^{\otimes k}$ by the subspace spanned by all pure tensors $v_{1} \otimes \cdots \otimes v_{k}$ that have $v_{i}=v_{j}$ for some distinct indices $i, j$. The image in $\bigwedge^{k} V$ of $v_{1} \otimes \cdots \otimes v_{k}$ for arbitrary vectors $v_{1}, \ldots, v_{k}$ is denoted by $v_{1} \wedge \cdots \wedge v_{k}$. It is non-zero if and only if the $v_{i}$ are linearly independent, and then for any other basis $w_{1}, \ldots, w_{k}$ of $\left\langle v_{1}, \ldots, v_{k}\right\rangle$ the element $w_{1} \wedge \cdots \wedge w_{k}$ is a scalar multiple of $v_{1} \wedge \cdots \wedge v_{k}$. In this sense, (images of) pure tensors modulo scalar factors bijectively represent $k$-dimensional subspaces of $V$.

Proof of Theorem 2.2 For $k=1, \ldots, n$ and $\pi \in S_{n}$ consider the morphism of algebraic varieties

$$
\Psi_{k, \pi}:\left(\bigwedge^{k-1} V\right)^{n} \times V^{n} \rightarrow\left(\bigwedge^{k} V\right)^{n},\left(\left(\omega_{j}\right)_{j},\left(u_{j}\right)_{j}\right) \mapsto\left(\omega_{j} \wedge u_{\pi(j)}\right)_{j}
$$

which models adding the $k$-th row $\left(u_{1}, \ldots, u_{n}\right)=\left(v_{k j}\right)_{j}$ of the array to the $n$-tuple of $(k-1)$-dimensional spaces constructed so far, according to the permutation $\pi$.

Let $D \subseteq V^{n}$ denote the hypersurface, defined by the determinant det $\in\left(V^{*}\right)^{\otimes n}$, consisting of all $n$-tuples of vectors that do not form a basis. Assuming that the characteristic of $K$ does not divide $\operatorname{ELS}(n)-\operatorname{OLS}(n)$, we will prove that for each $k=0, \ldots, n$ there exists a hypersurface $H_{k}$ in $\left(\bigwedge^{k} V\right)^{n}$, the Cartesian product of $n$ copies of the $k$-th exterior power of $V$, having the following two properties:

(1) each $H_{k}$ contains the set of tuples $\left(\omega_{1}, \ldots, \omega_{n}\right) \in\left(\bigwedge^{k} V\right)^{n}$ for which some $\omega_{i}$ is zero; and

(2) $H_{k-1}$ and $H_{k}$ are related by

$$
\bigcap_{\pi \in S_{n}}\left(\Psi_{k, \pi}^{-1} H_{k}\right) \subseteq\left(H_{k-1} \times V^{n}\right) \cup\left(\left(\bigwedge^{k-1} V\right)^{n} \times D\right)
$$


In other words, if for some $(\omega, u) \in\left(\bigwedge^{k-1} V\right)^{n} \times V^{n}$ it is true that for all $\pi \in S_{n}, \Psi_{k, \pi}(\omega, u)$ is in $H_{k}$, then either $\omega$ is already in $H_{k-1}$ or $u$ is a linearly dependent system (or both).

The latter condition implies that if $\left(\omega_{1}, \ldots, \omega_{n}\right) \in\left(\bigwedge^{k-1} V\right)^{n}$ lies outside $H_{k-1}$, then for any basis $u_{1}, \ldots, u_{n}$ of $V$ there exists a permutation $\pi$ such that the tuple $\left(\omega_{1} \wedge u_{\pi(1)}, \ldots, \omega_{n} \wedge u_{\pi(n)}\right)$ lies outside $H_{k}$.

The online algorithm is then as follows. Take any point $\left(\omega_{0,1}, \ldots, \omega_{0, n}\right) \in\left(\bigwedge^{0} V\right)^{n}$ outside $H_{0}$. After having constructed $\left(\omega_{k-1,1}, \ldots, \omega_{k-1, n}\right) \in\left(\bigwedge^{k-1} V\right)^{n} \backslash H_{k-1}$ for $k \geq 1$ read the $k$-th row $\left(v_{k 1}, \ldots, v_{k n}\right)$ of the array and choose $\pi_{k}$ such that $\left(\omega_{k j}\right)_{j}:=\Psi_{k, \pi_{k}}\left(\left(\omega_{j}\right)_{j},\left(v_{k j}\right)_{j}\right) \notin H_{k}$. This yields permutations $\pi_{1}, \ldots, \pi_{n}$ and an $n$ tuple of pure tensors $\left(\omega_{n, 1}, \ldots, \omega_{n, n}\right)$ that are all non-zero since the tuple lies outside $H_{n}$, and we are done.

It remains to construct the $H_{k}$. For this it is convenient to work with ordinary tensors first and then take the quotient to arrive at exterior powers. Thus consider

$$
\Phi_{k, \pi}:\left(V^{\otimes k-1}\right)^{n} \times V^{n} \rightarrow\left(V^{\otimes k}\right)^{n},\left(\left(\omega_{j}\right)_{j},\left(v_{j}\right)_{j}\right) \mapsto\left(\omega_{j} \otimes v_{\pi(j)}\right)_{j}
$$

and its co-morphism, regarded as a map

$$
\Phi_{k, \pi}^{*}:\left(\left(V^{*}\right)^{\otimes k}\right)^{\otimes n} \rightarrow\left(\left(V^{*}\right)^{\otimes k-1}\right)^{\otimes n} \otimes\left(V^{*}\right)^{\otimes n} .
$$

More explicitly, for numbers $a_{11}, \ldots, a_{k n} \in[n]$ we use the notation

$$
\left[\begin{array}{ccc}
a_{11} & \ldots & a_{1 n} \\
\vdots & & \vdots \\
a_{k 1} & \ldots & a_{k n}
\end{array}\right]
$$

for the tensor product $\left(x_{a_{11}} \otimes \cdots \otimes x_{a_{k 1}}\right) \otimes \cdots \otimes\left(x_{a_{1} n} \otimes \cdots \otimes x_{a_{k n}}\right)$, an element of $\left(\left(V^{*}\right)^{\otimes k}\right)^{\otimes n}$, where $x_{1}, \ldots, x_{n} \in V^{*}$ are the coordinate functions on $V=K^{n}$ dual to the standard basis $e_{1}, \ldots, e_{n}$. The linear map $\Phi_{k, \pi}^{*}$ sends (1) to

$$
\left[\begin{array}{ccc}
a_{11} & \ldots & a_{1 n} \\
\vdots & & \vdots \\
a_{k-1,1} & \ldots & a_{k-1, n}
\end{array}\right] \otimes\left[a_{k \pi^{-1}(1)} \ldots a_{k \pi^{-1}(n)}\right]
$$

Hence, the map $\sum_{\pi} \operatorname{sgn}(\pi) \Phi_{k, \pi}^{*}$ maps (1) to

$$
c \cdot\left[\begin{array}{ccc}
a_{11} & \ldots & a_{1 n} \\
\vdots & & \vdots \\
a_{k-1,1} & \ldots & a_{k-1, n}
\end{array}\right] \otimes \operatorname{det}, \text { where det }=\sum_{\pi \in S_{n}} \operatorname{sgn}(\pi)[\pi(1) \ldots \pi(n)]
$$

and where the scalar $c$ is 0 if the numbers $a_{k j} \in[n], j=1, \ldots, n$ are not all distinct, and equal to the sign of the permutation $j \mapsto a_{k j}$ otherwise. 
Let $\Theta_{k}:\left(\left(V^{*}\right)^{\otimes k}\right)^{\otimes n} \rightarrow\left(\left(V^{*}\right)^{\otimes k-1}\right)^{\otimes n}$ be the linear map that sends (1) to

$$
c \cdot\left[\begin{array}{ccc}
a_{11} & \ldots & a_{1 n} \\
\vdots & & \vdots \\
a_{k-1,1} & \ldots & a_{k-1, n}
\end{array}\right]
$$

without the determinantal factor. The natural projection $V^{\otimes k} \rightarrow \bigwedge^{k} V$ identifies, dually, the space of linear functions on $\bigwedge^{k} V$ with the space of alternating elements of $\left(V^{*}\right)^{\otimes k}$, i.e. tensors which change with $\operatorname{sgn}(\sigma)$ if a permutation $\sigma \in S_{k}$ is applied to the tensor factors. We take $F_{n}:=\operatorname{det}^{\otimes n}$ as defining equation for $H_{n} \subseteq\left(\bigwedge^{n} V\right)^{n}$. Define $F_{n-1}, \ldots, F_{0}$ inductively by $F_{k-1}:=\Theta_{k} F_{k}$. Each $F_{k}$ is an $n$-linear function on $\left(\bigwedge^{k} V\right)^{n}$, so that its zero set $H_{k}$ contains all $n$-tuples for which some entry is zero. Moreover, by construction a linear combination of the pull-backs $\Psi_{k, \pi}^{*} F_{k}=\Phi_{k, \pi}^{*} F_{k}$ over all $\pi$ equals $F_{k-1} \otimes$ det. This implies the second requirement on the $H_{k}$. The only thing that could go wrong is that $F_{k}=0$, in which case $H_{k}$ is the entire space rather than a hypersurface. Hence we need to verify that

$$
\Theta_{1} \circ \Theta_{2} \circ \cdots \circ \Theta_{n} F_{n} \neq 0 .
$$

In $F_{n}$ only tensors of the form

$$
\left[\begin{array}{ccc}
a_{11} & \ldots & a_{1 n} \\
\vdots & & \vdots \\
a_{n 1} & \ldots & a_{n n}
\end{array}\right]
$$

appear in which all columns are permutations of $[n]$, and then with coefficient equal to the product of the signs of these permutations. Moreover, $\Theta_{1} \cdots \Theta_{n}$ kills such a tensor unless each of its rows is also a permutation of $[n]$. Thus only Latin squares contribute to the expression above, and each Latin square contributes exactly its sign. Hence we find that

$$
\Theta_{1} \circ \cdots \circ \Theta_{n} F_{n}=\operatorname{ELS}(n)-\operatorname{OLS}(n),
$$

and this proves Theorem 2.2.

Remark 3.1 Of course $\Theta_{n} F_{n}$ is just some non-zero scalar multiple of a suitable determinant. Next we claim that $\Theta_{n-1} \Theta_{n} F_{n}$ is zero for odd $n$ and non-zero for even $n$. To prove both statements note that

$$
\left[\begin{array}{ccc}
a_{11} & \ldots & a_{1 n} \\
\vdots & & \vdots \\
a_{n-2,1} & \ldots & a_{n-2, n}
\end{array}\right]
$$


can have a non-zero coefficient in $\Theta_{n-1} \Theta_{n} F_{n}$ only if it can be extended to a tensor

$$
\left[\begin{array}{ccc}
a_{11} & \ldots & a_{1 n} \\
\vdots & & \vdots \\
a_{n 1} & \ldots & a_{n n}
\end{array}\right]
$$

in which the last two rows and all columns are permutations of [ $n$ ]. The coefficient of (3) in $\Theta_{n-1} \Theta_{n} F_{n}$ is then the product of these $n+2$ signs, summed over all possible extensions. For odd $n$ interchanging the last two rows gives a sign-reversing involution on the set of extensions, hence $\Theta_{n-1} \Theta_{n} F_{n}$ is zero, as claimed. For $n$ even consider, for instance, the tensor

$$
\left[\begin{array}{cccc}
1 & 2 & \ldots & n \\
2 & 3 & \ldots & 1 \\
\vdots & \vdots & & \vdots \\
n-2 & n-1 & \ldots & n-3
\end{array}\right]
$$

It has two possible extensions, with last two rows equal to

$$
\left[\begin{array}{cccc}
n-1 & n & \ldots & n-2 \\
n & 1 & \ldots & n-1
\end{array}\right] \text { and }\left[\begin{array}{cccc}
n & 1 & \ldots & n-1 \\
n-1 & n & \ldots & n-2
\end{array}\right]
$$

respectively. The product of the 2 row signs is the same for both extensions, and the product of the $n$ column signs is multiplied by $(-1)^{n}$, which is an even power. Hence the tensor above has a coefficient with absolute value 2 in $\Theta_{n-1} \Theta_{n} F_{n}$. This means that $H_{n-2}$ is a proper hypersurface (provided that char $K \neq 2$ ). With a bit more effort, using the fact that the Grassmannian of $(n-2)$-subspaces of $V$ is cut out from $\bigwedge^{n-2} V$ by quadratic polynomials one can show that $H_{n-2}$ does not contain all $n$-tuples of pure $(n-2)$-tensors, so that a sufficiently general $n$-tuple of $(n-2)$-spaces can be extended with the last two rows. In particular, this readily implies that given a $3 \times n$-array whose rows are bases of $K^{n}$ with $n$ even and $\geq 4$, there are always three permutations leading to an array in which the columns are independent triples.

A similar argument shows the following.

Proposition 3.2 Let $\ell, m$ be natural numbers with $\ell>1$ and set $n:=\ell \cdot m$. If the characteristic of $K$ does not divide $\operatorname{ELS}(m)-\operatorname{OLS}(m)$, then there exists an online algorithm that sequentially takes $m+1$ bases of $V=K^{n}$ as input and immediately after reading the $i$-th basis arranges it as the $i$-th row in an $(m+1) \times n$-array such that each column of the array eventually consists of $m+1$ linearly independent vectors.

Proof As in the previous remark, it suffices to show that $F_{n-m}$ is non-zero, and for this it suffices to exhibit a basis vector in $\left(\left(V^{*}\right)^{\otimes(n-m)}\right)^{\otimes n}$ that has a non-zero coefficient in $F_{n-m}$. For this, take any basis vector of the form

$$
\left[\begin{array}{llll}
A_{1} & A_{2} & \ldots & A_{\ell}
\end{array}\right]
$$


where all columns of $A_{j}$ contain exactly the $n-m$ numbers $\{1, \ldots, n\} \backslash\{(j-1) m+$ $1, \ldots, j m\}$. The coefficient in $F_{n-m}$ is the sum, over all extensions to $n \times n$-arrays in which all the last $m$ rows and all columns are permutations, of the products of their $n+m$ signs. Up to a sign, this is just $(\operatorname{ELS}(m)-\mathrm{OLS}(m))^{\ell}$, hence non-zero in $K$ by assumption.

For some values of $n$ this proposition gives results sharper than those in [10], but only for matroids representable over fields of characteristic zero or sufficiently large.

\section{Odd dimensions}

In this section we prove Theorem 2.3. So we assume that $n$ is odd and that $K$ contains primitive $m$-th roots of unity for all odd $m \leq n$, and we will show that no online algorithm exists for arranging the basis vectors.

Proof of Theorem 2.3 We argue that we can force any online algorithm for choosing the row permutations into making an error. For this, we first feed the algorithm $n-2$ times the standard basis $e_{1}, \ldots, e_{n}$. If it does not make an error yet, then it will arrange these bases as the rows of an $(n-2) \times n$-array in which each column consists of $n-2$ distinct standard basis vectors. For $l=1, \ldots, n$ let $V_{l}$ denote the space spanned by the vectors in the $l$-th column.

Now we construct a graph with vertex set $[n]$ and an edge $\{i, j\}$ for every $l$ with $e_{i}, e_{j} \notin V_{l}$. This graph has $n$ edges, some of which may be double, as some of the $V_{l}$ may coincide. Each $e_{i}$ is missing from exactly two of the $V_{l}$, so the graph is regular of degree 2 and hence a union of cycles. Since $n$ is odd, the graph has an $m$-cycle for some odd $m \leq n$. Without loss of generality, we may assume that for $l=1, \ldots, m-1$ the space $V_{l}$ misses exactly $e_{l}$ and $e_{l+1}$ and that $V_{m}$ misses exactly $e_{m}$ and $e_{1}$.

We produce an $(n-1)$-st basis $v_{1}, \ldots, v_{n}$ for the algorithm to arrange, as the columns of the following block matrix:

$$
\left[\begin{array}{ll}
Y & 0 \\
0 & Z
\end{array}\right] \text {, }
$$

where $Y$ has size $m \times m$ and $Z$ has size $(n-m) \times(n-m)$, and where

$$
Y=\left[\begin{array}{cccc}
\zeta & \zeta^{2} & \ldots & \zeta^{m} \\
\zeta^{2} & \zeta^{4} & \ldots & \zeta^{2 m} \\
\vdots & \vdots & & \vdots \\
\zeta^{m} & \zeta^{2 m} & \ldots & \zeta^{m^{2}}
\end{array}\right]
$$

with $\zeta$ a primitive $m$-th root of unity, so that $\operatorname{det} Y$ is non-zero. It does not matter what $Z$ is, as long as it is also invertible, so that $v_{1}, \ldots, v_{n}$ are legitimate input to the algorithm.

Since $V_{m+1}, \ldots, V_{n}$ already contain the vectors $v_{1}, \ldots, v_{m}$, the latter must be assigned in some order to $V_{1}, \ldots, V_{m}$. Suppose that $V_{l}$ gets the $\pi(l)$-th vector $v_{\pi(l)}$, 
where $\pi \in S_{n}$ stabilises the sets $[m]$ and $\{m+1, \ldots, n\}$. Set $V_{l}^{\prime}:=V_{l} \oplus\left\langle v_{\pi(l)}\right\rangle$, where the sum is, indeed, direct if the algorithm makes no error yet.

For $l=1, \ldots, m$, let $z_{l} \in V^{*}$ be a normal vector of the space $V_{l}^{\prime}$. Thus $z_{l}$ annihilates all standard basis vectors except $e_{l}$ and $e_{l+1}$ (index modulo $m$ with offset 1), and is hence of the form $a x_{l}+b x_{l+1}$. The scalars $a$ and $b$ are determined by the fact that $z_{l}$ also annihilates the vector with first $m$ entries $\zeta^{i \cdot \pi(l)}, i=1, \ldots, m$. Thus we find that $a \zeta^{l \cdot \pi(l)}+b \zeta^{(l+1) \cdot \pi(l)}=0$, and we can choose

$$
z_{l}=\zeta^{\pi(l)} x_{l}-x_{l+1}
$$

We claim that $z_{1}, \ldots, z_{m}$ are linearly dependent. To see this, we compute the determinant of their coefficients with respect to $x_{1}, \ldots, x_{m}$ :

$$
\operatorname{det}\left[\begin{array}{ccccc}
\zeta^{\pi(1)} & & & & -1 \\
-1 & \zeta^{\pi(2)} & & & \\
& -1 & \ddots & & \\
& & \ddots & \zeta^{\pi(m-1)} & \\
& & & -1 & \zeta^{\pi(m)}
\end{array}\right]=\zeta^{\pi(1)+\pi(2)+\cdots+\pi(m)}-1
$$

Here the minus sign is in fact independent of the parity of $m$ : for $m$ odd, the $m$-cycle is an even permutation but the number of minus signs is odd, while for $m$ even, the $m$-cycle is odd but the number of minus signs is even. Now the exponent equals

$$
\pi(1)+\pi(2)+\cdots+\pi(m)=1+2+\cdots+m=\frac{1}{2} m(m+1) .
$$

Since $m$ is odd, the latter number is a multiple of $m$, and hence the determinant is zero.

Since $z_{1}, \ldots, z_{m}$ are linearly dependent, we find that $V_{1}^{\prime} \cap \cdots \cap V_{m}^{\prime}$ has co-dimension strictly larger than $m$. Then $V_{1}^{\prime} \cap \cdots \cap V_{n}^{\prime}$ has positive dimension. If we choose, for the $n$-th basis, any basis containing a vector in that intersection, the algorithm is forced to make an error. This concludes the proof of Theorem 2.3.

Remark 4.1 For $n=3$, the above can be shown to be the only counterexample to $\mathrm{OBC}_{3}$ up to symmetries, by a computation similar to the one in [5, Proof of Theorem 2]. So $\mathrm{OBC}_{3}(K)$ holds for fields $K$ not containing primitive cube roots of unity, such as $K=\mathbb{R}$. We did not study the validity of $\mathrm{OBC}_{n}(\mathbb{R})$ for other odd values of $n$.

We conclude this section with a relation between the generalisation from [16] of $\mathrm{ATLSC}_{n}$ to odd $n$ and a special case of $\mathrm{OBC}_{n}$. A different relation between a variant of ATLSC and a weakening of RBC for odd $n$ was established in [1].

Proposition 4.2 Consider only $n \times n$ Latin squares that have all diagonal entries equal to the symbol $n$. If the number of even Latin squares minus the number of odd Latin squares among these is non-zero when regarded as an element of $K$, then there exists an online algorithm that sequentially takes $n$ bases of $V=K^{n}$, each of which contains 
the standard basis vector $e_{n}$, and arranges these as the rows of an $n \times n$-array whose columns are also bases.

Proof Write $W:=K^{n-1}$, so that $V=W \oplus\left\langle e_{n}\right\rangle$. The algorithm will first replace each basis vector unequal to $e_{n}$ by its projection in $W$. The proof is now identical to that of Theorem 2.2, except that in the $k$-th row we put the copy of $e_{n}$ on position $k$. Hence for each $k \in[n]$ and every $\pi \in S_{n}$ with $\pi(k)=k$ we define

$$
\begin{aligned}
& \Phi_{k, \pi}:\left(V^{\otimes k-1}\right)^{n} \times W^{[n] \backslash\{k\}} \rightarrow\left(V^{\otimes k}\right)^{n}, \\
& \left(\left(\omega_{j}\right)_{j},\left(w_{j}\right)_{j}\right) \mapsto\left(\omega_{1} \otimes w_{\pi(1)}, \ldots, \omega_{k} \otimes e_{n}, \ldots, \omega_{n} \otimes w_{\pi(n)}\right) .
\end{aligned}
$$

Then $\Phi_{k, \pi}^{*}$ maps a tensor (1) to 0 if $a_{k k} \neq n$ or if $a_{k j}=n$ for some $j \neq k$, and to

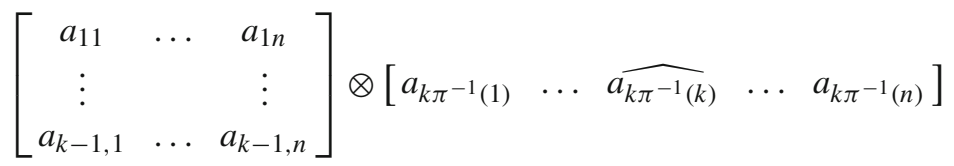

(with $\hat{\cdot}$ indicating that that factor is left out) otherwise. Consequently, the linear combination $\sum_{\pi \in S_{n}: \pi(k)=k} \operatorname{sgn}(\pi) \Phi^{*}$ maps a tensor (1) to

$$
c \cdot\left[\begin{array}{ccc}
a_{11} & \ldots & a_{1 n} \\
\vdots & & \vdots \\
a_{k-1,1} & \ldots & a_{k-1, n}
\end{array}\right] \otimes \operatorname{det}_{n-1}
$$

where

$$
\operatorname{det}_{n-1}=(-1)^{n-k} \sum_{\sigma \in S_{n-1}} \operatorname{sgn}(\sigma)\left[\begin{array}{lll}
\sigma(1) & \ldots & \sigma(n-1)
\end{array}\right]
$$

and where $c=\operatorname{sgn}(\tau)$ if the map $\tau: j \mapsto a_{k j}$ is a permutation of $[n]$ with $\tau(k)=n$, and $c=0$ otherwise. The factor $(-1)^{n-k}$ in $\operatorname{det}_{n-1}$ accounts for the relation $\pi=$ $(k, k+1, \ldots, n) \tau$. Let

$$
\Theta_{k}:\left(\left(V^{*}\right)^{\otimes k}\right)^{\otimes n} \rightarrow\left(\left(V^{*}\right)^{\otimes k-1}\right)^{\otimes n}
$$

be the linear map taking (1) to the previous expression without the factor $\operatorname{det}_{n-1}$. Starting with $F_{n}$ as in Sect. 3 and defining $F_{k-1}:=\Theta_{k} F_{k}$, we find that $F_{0}$ equals the sum of the signs of all Latin squares in the statement of the proposition.

\section{Further remarks}

Both ATLSC and RBC are notoriously difficult conjectures, and our results do not shed new light on these conjectures. But our results do lead to a number of problems that might be more tractable than ATLSC and RBC themselves. 
First, our construction in the previous section showed that for odd $n$ any online algorithm can be forced to make an error in the very last row. Are there examples where the algorithm is forced to make an error earlier? Or, on the contrary, can one formulate a combinatorial conjecture à la ATLSC which, using algebro-geometric techniques, would imply the existence of an online algorithm for arranging any $(n-1) \times n$-array?

Second, one can try to formulate an online version of Rota's basis conjecture for general matroids, where the algorithm does not know the entire matroid on $n^{2}$ elements in advance, but rather the matroid structure is disclosed row by row and the algorithm is required to arrange the rows immediately when they become available. The previous section shows that this generalisation fails for odd rank. But in fact, it fails for any $n \geq 3$, for the following simple reason. First consider the uniform matroid $M$ of rank $n$ on $(n-1) n$ elements, arranged in an $(n-1) \times n$-array $\left(e_{i j}\right)_{i j}$, whose rows are of course bases. Since this matroid is preserved by the entire group $\left(S_{n}\right)^{n-1}$ of row permutations, we may assume that the algorithm leaves each $e_{i j}$ in place. Then one can verify that $M$ has a one-element extension $M \cup\{e\}$ in which the only dependent sets of size $n$ are $\left\{e_{i j} \mid i=1, \ldots, n-1\right\} \cup\{e\}$ for $j=1, \ldots, n$; here we use that $n>2$. If the $n$-th basis that we give as input to the algorithm contains an $e$ such that $M \cup\{e\}$ has this structure, then it is forced to make an error. These counterexamples can, in fact, be realised as linear matroids over suitable fields. The difference with $\mathrm{OBC}_{n}$ is that the algorithm gets only the matroid as input, not its realisation.

So $\mathrm{OBC}_{n}$ does not have a meaningful direct generalisation to $n \times n$-arrays. However, we think that the following problem (which asks for an online variant of [10]) may well be tractable.

Problem 5.1 Given $n$, what is the maximal value of $k$ such that there exists an online algorithm that on sequential input of $k$ bases of a rank- $n$ matroid arranges each basis immediately as the next row in a rectangular table, in such a way that in the resulting $k \times n$-table the columns are independent sets?

Third, a generalisation of $\mathrm{RBC}_{n}$ that we learned from a referee concerns an $n \times n$ array of vectors in a vector space of dimension $d \geq n$. It states that if each row consists of $n$ independent vectors, then the vectors can be permuted within their rows such that afterwards each column consists of $n$ independent vectors, as well. The Latin square analogue of this generalisation was proved in [5, Theorem 1], just before Galvin proved the stronger Dinitz conjecture [8]. For $d=n$ this generalisation is just $\mathrm{RBC}_{n}$. Conversely, if the field is infinite (or sufficiently large), then any instance of the generalisation can be transformed into an instance of $\mathrm{RBC}_{n}$ by choosing a projection into $n$-space that keeps the rows independent. So the generalisation is equivalent to $\mathrm{RBC}_{n}$ and follows from $\mathrm{ATLSC}_{n}$, again for sufficiently large fields. However, the reduction via a projection does not settle the online status of this generalisation, since to choose the projection one needs to know at least the spans of each of the $n$

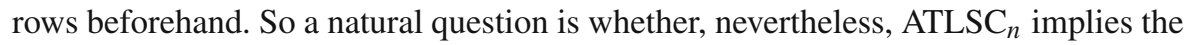
existence of an online algorithm for this generalisation.

Acknowledgments J.D. is supported by a Vidi Grant from the Netherlands Organisation for Scientific Research (NWO). 


\section{References}

1. Aharoni, R., Kotlar, D.: A weak version of Rota's basis conjecture for odd dimensions. SIAM J. Discrete Math. 2011. To appear, preprint at arXiv:1110.1830

2. Alon, N., Tarsi, M.: Colorings and orientations of graphs. Combinatorica 12(2), 125-134 (1992)

3. Berndsen, J.: Three problems in algebraic combinatorics. Master's thesis, Eindhoven University of Technology (2012). Available electronically at http://alexandria.tue.nl/extra1/afstversl/wsk-i/ berndsen 2012

4. Chan, W.: An exchange property of matroid. Discrete Math. 146, 299-302 (1995)

5. Chow, T.Y.: On the Dinitz conjecture and related conjectures. Discrete Math. 145(1-3), 73-82 (1995)

6. Drisko, A.A.: On the number of even and odd Latin squares of order $p+1$. Adv. Math. 128(1), 20-35 (1997)

7. Drisko, A. A.: Proof of the Alon-Tarsi conjecture for $n=2^{r} p$. Electron. J. Combin. 5, 1998. Research paper $28,5 \mathrm{pp}$

8. Galvin, F.: The list chromatic index of a bipartite multigraph. J. Comb. Theory Ser. B 63(1), 153-158 (1995)

9. Geelen, J., Humphries, P.J.: Rota's basis conjecture for paving matroids. SIAM J. Discrete Math. 20(4), 1042-1045 (2006)

10. Geelen, J., Webb, K.: On Rota's basis conjecture. SIAM J. Discrete Math. 21(3), 802-804 (2007)

11. Glynn, D.G.: The conjectures of Alon-Tarsi and Rota in dimension prime minus one. SIAM J. Discrete Math. 24(2), 394-399 (2010)

12. Huang, R., Rota, G.-C.: On the relations of various conjectures on latin squares and straightening coefficients. Discrete Math. 128, 225-236 (1994)

13. Jeannette, C.M.: Janssen. On even and odd Latin squares. J. Combin. Theory Ser. A 69(1), 173-181 (1995)

14. Onn, S.: A colorful determinantal identity, a conjecture of Rota, and Latin squares. Am. Math. Mon. 104(2), 156-159 (1997)

15. Wild, M.: On Rota's problem about $n$ bases in a rank $n$ matroid. Adv. Math. 108(2), 336-345 (1994)

16. Zappa, P.: The Cayley determinant of the determinant tensor and the Alon-Tarsi conjecture. Adv. Appl. Math. 19(1), 31-44 (1997) 